\title{
Una alternativa multicriterio a la valoración de empresas: aplicación a las Cajas de Ahorro
}

\author{
JERÓNIMO AZNAR BELLVER \\ Departamento de Economía y Ciencias Sociales, UNIVERSIDAD POLITÉCNICA DE \\ VALENCIA, ESPAÑA. E-mail: jaznar@esp.upv.es
}

ROBERTO CERVELLÓ ROYO

Departamento de Economía y Ciencias Sociales, UNIVERSIDAD POLITÉCNICA DE

VALENCIA, ESPAÑA. E-mail: rocerro@esp.upv.es

\section{FERNANDO GARCÍA GARCÍA}

Departamento de Economía y Ciencias Sociales, UNIVERSIDAD POLITÉCNICA DE VALENCIA, ESPAÑA. E-mail: fergarga@esp.upv.es

\begin{abstract}
RESUMEN
En numerosas ocasiones las metodologías de valoración usualmente empleadas presentan dificultades en su aplicación a la valoración de empresas, bien porque el valorador no dispone de suficientes datos para utilizar los métodos comparativos tradicionales, bien por la dificultad de estimar los flujos de caja futuros con objetividad. Ante esta problemática, el presente trabajo propone una alternativa que combina el método multicriterio CRITIC con el Ratio de Valoración de las Normas Internacionales de Valuación. La nueva metodología se inserta dentro de los métodos comparativos o de enfoque de mercado y requiere únicamente disponer de un número reducido de empresas comparables. En este trabajo se ha aplicado a la valoración de una caja de ahorros. La valoración de este tipo de instituciones financieras, por la situación actual del sector, es un cometido de gran interés y constituye además un ejemplo muy ilustrativo para mostrar las fortalezas de la metodología propuesta.
\end{abstract}

Palabras clave: Valoración de empresas; CRITIC; Ratio de Valoración; Cajas de Ahorro

\section{A Multicriteria Alternative to Companies’ Valuation: Application to a Spanish Savings Bank}

\begin{abstract}
In several occasions the traditional valuation methodologies present difficulties in their application to the companies' valuation, either due to valuator's lack of enough data to use in the traditional comparative methods, or due to the difficulty when estimating the future cash-flows with objectivity. In this situation, the present study suggests an alternative which combines the CRITIC multicriteria methodology with the Valuation Ratio of the International Standards of Valuation. The new methodology is included in the comparative or market approach methods and it only requires having at its disposal a small number of comparable companies. In this work this methodology is applied to get the value of a savings bank. The valuation of this kind of financial institutions, due to the current situation of the sector, implies a task of great interest and it also constitutes a very illustrative example to show the strengths of the proposed methodology.
\end{abstract}

Keywords: Companies Valuation; CRITIC; Valuation Ratio; Savings Banks

Clasificación JEL: C44, G1

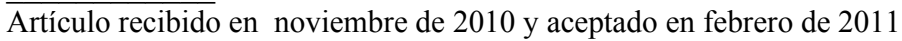

Artículo disponible en versión electrónica en la página www.revista-eea.net, ref. ə-29105 


\section{INTRODUCCIÓN}

La valoración de empresas es una actividad de gran relevancia en el contexto económico actual. Conocer el valor de una empresa es importante para los gestores de la misma, ya que de esta forma pueden observar el impacto que sus decisiones tienen sobre la creación de valor en la empresa, pudiendo establecer un modelo de evaluación de su propia gestión empresarial. Por otro lado, el conocimiento del valor es relevante para accionistas e inversores, ya que así pueden abordar de forma más adecuada determinadas operaciones societarias, como ampliaciones de capital, fusiones, adquisiciones, ofertas públicas de valores, financiación de inversiones, entre otras. Por último el conocimiento del valor de las empresas es fundamental en los casos de herencias o donaciones.

En las Normas Internacionales de Valuación (International Valuation Standards Committee -IVSC, 2007) se clasifican los métodos de valoración en tres grandes grupos: los comparativos o de enfoque de mercado, los de actualización de rentas y los del coste. Dentro de ellos existen distintas orientaciones metodológicas para la valoración de empresas: la valoración patrimonial, el descuento de los flujos de caja libres, los métodos compuestos a partir de los anteriores, los modelos econométricos o de regresión, las opciones reales, o la valoración analógico-bursátil.

Todos estos métodos tradicionales, a pesar de su indudable utilidad, presentan una serie de limitaciones que afectan a la práctica valorativa:

1) Algunos de los métodos comparativos, como el análisis de regresión, necesitan de una amplia base de datos de activos comparables. En numerosos casos no se dispone de una base de datos suficientemente amplia y en el caso de la valoración de empresas este problema es habitual. (Moya, 1995; Miralles y Miralles, 2002; Vidal et al., 2004; García et al. 2008).

2) En la metodología de actualización de rentas, se trabaja con datos que deben estimarse previamente, ya que se basa en prever la evolución futura del activo a valorar. En el caso de la valoración de empresas supone determinar los flujos de caja futuro y su valor residual y aplicar una tasa de descuento adecuada. Evidentemente, estas previsiones introducen una elevada carga de subjetividad en las valoraciones, que son muy sensibles ante modificaciones en los escenarios futuros planteados.

3) Los métodos del coste son metodologías de valoración que se aplican exclusivamente a edificios y suelo urbano y, obviamente, no son extrapolables a la valoración de empresas, aunque se podría considerar la valoración patrimonial como afín a esta metodología.

4) Por último, en todas las metodologías de valoración tradicionales, resulta complicado introducir directamente variables cualitativas en el proceso valorativo. Esto supone una limitación seria, pues resulta innegable la 
importancia que tienen en el valor de una empresa aspectos como el liderazgo comercial, la profesionalidad del equipo humano, el prestigio de la empresa, el posicionamiento internacional, etc.

Distintos trabajos proponen soluciones a este tipo de limitaciones mediante la utilización de metodologías alternativas provenientes del campo de la decisión multicriterio. En ese sentido encontramos aplicaciones a la valoración de la programación por metas (Aznar y Guijarro, 2004, 2007 a y 2007 b), del proceso analítico jerárquico (Aznar y Estruch, 2007), del proceso analítico en red (Aragones-Beltran et al.2008, Garcia-Melón et al, 2008) y de la conjunción de varias de estas técnicas (Aznar et al., 2007, 2008; Cervelló et al. 2010; Guijarro y Guijarro, 2010).

En este trabajo se propone un nuevo modelo de valoración compuesto por CRITIC y el Ratio de Valoración al que denominamos CRITICRatio. Este modelo queda ubicado dentro de los métodos comparativos, que como es sabido calculan el valor de un activo mediante su comparación con activos comparables de los cuales conocemos sus características y su precio. En el caso de la valoración de empresas calcularemos el valor desconocido de una empresa comparándolo con otras empresas parecidas a ella de las que sí se conoce su valor, por ejemplo, por cotizar en el mercado bursátil, y la comparación se realiza utilizando una serie de criterios que explican el valor de ese tipo de empresas.

La metodología que proponemos está especialmente indicada para valoraciones de empresas en las que el número de comparables es reducido y se trabaja con datos disponibles de la contabilidad empresarial. Además permite la inclusión de variables cualitativas mediante la combinación con AHP, aunque en este trabajo no se va a abordar este aspecto.

El resto del trabajo se articula de la siguiente manera. El siguiente epígrafe se dedica a presentar detalladamente la nueva metodología de valoración. El epígrafe tres expone un caso de estudio en el que la nueva metodología se aplica a la valoración de una caja de ahorros española, la Caja de Ahorros del Mediterráneo (CAM). El epígrafe cuatro recoge las principales conclusiones del trabajo.

\section{MODELO DE VALORACIÓN CRITICRATIO}

El modelo de valoración que proponemos se compone de los métodos CRITIC (Diakauloki et al, 1995) y del Ratio de Valoración (IVSC, 2007), y consta de los siguientes pasos:

Primer Paso. Selección de comparables

Segundo Paso. Selección de los criterios explicativos del valor

Tercer Paso. Ponderación de los criterios mediante CRITIC 
Cuarto Paso. $\quad$ Ponderación de las empresas

Quinto Paso. Cálculo del Ratio de Valoración

Sexto Paso. Cálculo del valor de la empresa objetivo

A continuación, se describe con mayor detalle cada uno de los pasos que componen el proceso de valoración.

Primer Paso. Selección de comparables.

Seleccionada la empresa a valorar, el primer punto es determinar las empresas comparables, que deben ser similares y, por lo tanto, comparables a la empresa que se desea valorar. Además, debe tratarse de empresas de las cuales conocemos su valor, por ejemplo, porque cotizan en el mercado bursátil.

Segundo Paso. Selección de los criterios explicativos del valor

Se seleccionan los criterios a emplear en el proceso de comparación y se elabora la base de datos. Como se ha comentado, la metodología de valoración propuesta se basa en la comparación de empresas. A partir de esa comparación, y conociendo el valor económico de las empresas comparables o testigo, se extrae el valor de la empresa problema. Resulta por tanto fundamental determinar en base a qué variables se va a realizar dicha comparación. En la literatura de valoración de empresas se emplean principalmente variables económico financieras extraídas de la contabilidad. El uso de este tipo de variables está muy extendido, no sólo en el campo de la valoración de empresas, sino también en campos tan diversos como el análisis del riesgo de crédito (Beaver (1966,1968), Altman (1968, 1971, 1973, 1993), Ohlson (1980), Sun and Shenoy (2007), Wang and Lee (2008), Psillaki et al (2010), Li et al. (2010)), el análisis de la performance empresarial (Yeh (1996), Halkos y Salamouris (2004), Malhotra (2009)) o la elaboración de rankings de empresas (Feng and Wang (2001), Deng et al. (2000), Garcia et al. (2010a)), por citar sólo algunos trabajos. En estos trabajos se valen de metodologías tan diversas como los tests de clasificación dicotómicos, el análisis discriminante, el análisis factorial, modelos logit y probit, artificial neuronal networks, DEA o TOPSIS.

Tercer Paso. Ponderación de los criterios mediante CRITIC

Se pondera el peso o importancia de los distintos criterios, aplicando CRITIC. No parece razonable pensar que todas las variables o criterios seleccionados tienen la misma importancia o influencia a la hora de determinar el valor empresarial. Resulta por lo tanto necesario asignar, de forma objetiva, una ponderación a cada uno de los criterios seleccionados en el paso anterior. 
CRITIC (Criteria Importance Through Intercriteria Correlation) (Diakoulaki et al., 1995) es un método de ponderación de criterios que define la importancia de los mismos mediante (1), a partir de sus valores normalizados por el rango.

$$
\mathrm{w}_{\mathrm{j}}=S_{\mathrm{j}} * \sum_{j=1}^{n} \sum\left(1-r_{j k}\right)
$$

siendo

$w_{j}=$ peso o ponderación del criterio $j$

$s_{j}=$ desviación típica del criterio $j$

$r_{j, k}=$ Coeficiente de correlación entre los criterios $j$ y $k$

Los pesos obtenidos $\left(w_{j}\right)$ se normalizan por la suma.

Aplicando CRITIC, un criterio tiene mayor peso cuanto mayor sea su desviación típica y menor correlación mantiene con los otros criterios.

Cuarto Paso. Ponderación de las empresas

Obtenido el peso $w_{j}$ de cada uno de los criterios se procede al cálculo de la ponderación de las distintas empresas mediante (2):

$$
\mathrm{x}_{\mathrm{i}}=\sum_{j=1}^{n} \mathrm{w}_{\mathrm{j}} \times C_{i j}
$$

donde

$x_{i}$ es la ponderación de la empresa $i$,

$w_{j}$ es el peso del criterio $j$,

$c_{i, j}$ es el valor del criterio $j$ para la empresa $i$

Quinto Paso. Cálculo del Ratio de Valoración

El Ratio de Valoración es una metodología propuesta en las Normas Internacionales de Valuación (1997) siendo su expresión matemática (3). En nuestro caso, el numerador es la suma de los valores de las empresas comparables u otro tipo de parámetro ligado al mismo y el denominador es la suma de las ponderaciones de las empresas comparables obtenidas en el paso anterior (cuarto paso).

$$
R V=\frac{\sum_{i=1}^{n} V_{i}}{\sum_{i=1}^{n} x_{i}}
$$

siendo 
$R V=$ Ratio de Valoración

$V_{i}=$ Valor de la empresa $i$

$x_{i}=$ Peso de la empresa obtenido con CRITIC

El Ratio de Valoración nos indica el valor de las empresas por unidad de ponderación.

Sexto Paso. Cálculo del valor de la empresa objetivo

El valor de la empresa objetivo se calcula mediante el producto del ratio obtenido en (3) por la ponderación de la empresa a valorar obtenida al aplicar (2).

\section{CASO DE ESTUDIO}

En este epígrafe se aplica la metodología propuesta a la valoración de una caja de ahorros de características comparables con algunas entidades financieras que cotizan en el mercado bursátil español. El hecho de que se haya seleccionado como empresa a valorar una caja de ahorros no es arbitrario. En la actualidad, como consecuencia de la crisis financiera internacional, el sistema financiero español está experimentando profundos cambios. Entre los cambios más significativos cabe destacar los procesos de fusión de cajas de ahorro. Y, en el futuro, no se puede descartar que estas entidades financieras, que actualmente tienen una forma jurídica peculiar, no tienen accionistas y, evidentemente, no cotizan en bolsa, se transformen en bancos con capital privado. De hecho, son muchos los organismos internacionales como el IMF, los que abogan por un cambio de la legislación española en este sentido. En el caso de que se diera esta evolución en el sector hacia la privatización, por ejemplo mediante ofertas públicas de valores, pensamos que la metodología presentada en este trabajo sería de gran ayuda a los valoradores que debieran determinar el valor de las instituciones financieras y, por ende, el precio de salida de las acciones.

Primer Paso. Selección de comparables.

Para realizar la valoración se eligieron como comparables aquellos bancos españoles que cotizan en la bolsa de valores y cuyo tamaño y cifra de negocios son similares a los de la empresa a valorar (Banco Pastor, Bankinter, Banco Sabadell, Banesto y Banco Popular). Al ser pocos los comparables disponibles, resulta un caso idóneo para aplicar CRITICRatio.

La caja de ahorros a valorar es la Caja de Ahorros del Mediterráneo (CAM), cuyo origen data de 1875 y que ha sido la primera caja de ahorros española en emitir cuotas participativas. Por sus características en cuanto a valor del activo (71.441.621 miles de euros) y beneficios (276.547 miles de euros), se trata de 
una empresa comparable con varias entidades financieras cotizadas, como se desprende de la tabla 1.

Tabla 1.

Activo total y beneficio a 31/12/2009

\begin{tabular}{|c|c|c|c|c|c|}
\hline & Pastor & Bankinter & Sabadell & Banesto & Popular \\
\hline $\begin{array}{c}\text { Activos totales } \\
\text { (miles } € \text { ) }\end{array}$ & 32.325 .235 & 54.467 .584 & 82.822 .886 & 126.220 .639 & 129.290 .148 \\
\hline $\begin{array}{c}\text { Beneficio } \\
\text { después de } \\
\text { impuestos } \\
\text { (miles } € \text { ) }\end{array}$ & 102.591 & 254.404 & 526.309 & 558.824 & 780.347 \\
\hline
\end{tabular}

Segundo Paso. Selección de los criterios explicativos del valor

Como ya se ha comentado anteriormente, la selección de las variables económico-financieras que servirán de criterios para llevar a cabo la comparación de empresas es un paso clave. Estas variables deben servir para caracterizar la gestión de las empresas y el grado de éxito o fracaso obtenido a raíz de la misma. Aunque no existe en la literatura una lista definida de los ratios contables que deben utilizarse, sí es fácil encontrar multitud de estudios que utilizan dicha información para analizar la performance empresarial, incluida la performance de las cajas de ahorro. Entre estos trabajos podemos destacar los de Kumbhakar et al. (2001), Prior (2003) y García-Cestona y Surroca (2006). Otros estudios que emplean igualmente información económico financiera se centran en la valoración de cajas de ahorro (Moya, 1996), o la elaboración de rankings de cajas de ahorro (Arévalo-Quijada et al. 2002; García et al. 2010b).

A partir del análisis de estos trabajos previos se ha podido establecer que todos los ratios utilizados se pueden agrupar en diversas categorías. O dicho de otro modo: existen ciertas dimensiones de la estructura económico financiera que resultan indispensables a la hora de caracterizar a una entidad financiera. De forma recurrente aparecen las dimensiones: inputs, outputs y gestión del riesgo, cuyos ratios más representativos son:

- Dimensión Inputs: Coste del Trabajo (Gasto de personal/Plantilla), Coste del Capital Físico (Amortización/Activo material) y Coste de los Depósitos/Capital.

- Dimensión Outputs: ROA (Resultado del ejercicio/Activo total) y Rentabilidad obtenida por el dinero prestado (Intereses y rendimientos asimilados/Inversiones crediticias). 
- Dimensión Gestión del riesgo: Morosidad, Fondo de Cobertura y Ratio BIS (ratio de solvencia).

Hay que tener en cuenta que con el objeto de cumplir con el principio de "a mayor, mejor" se ha calculado la inversa tanto de los criterios recogidos en el grupo de inputs como para la tasa de morosidad

Los valores de la media y la desviación típica de los ratios financieros de las empresas empleadas en la valoración aparecen en la tabla 2.

Tabla 2.

Valores medios y desviación típica de los ratios financieros para el ejercicio 2009

\begin{tabular}{|l|r|r|}
\hline & Media & $\begin{array}{c}\text { Desviación } \\
\text { Típica }\end{array}$ \\
\hline INPUTS & & \\
\hline Trabajo & 66,503 & 8,288 \\
\hline Coste del capital físico & 0,128 & 0,126 \\
\hline Coste de los depósitos/capital & 0,018 & 0,009 \\
\hline OUTPUTS & & \\
\hline ROA & 0,005 & 0,001 \\
\hline Rentabilidad obtenida por el dinero prestado & 0,042 & 0,018 \\
\hline TASAS & & \\
\hline Tasa de Morosidad & 0,039 & 0,010 \\
\hline Fondo de Cobertura & 0,744 & 0,233 \\
\hline Ratio BIS & 0,113 & 0,010 \\
\hline
\end{tabular}

Tercer Paso. Ponderación de los criterios mediante CRITIC

Con CRITIC, se determina la ponderación de cada uno de los criterios. En primer lugar, se procede a la normalización de las variables mediante el rango, para posteriormente calcular la desviación estándar de cada parámetro y la matriz de correlaciones. Los pesos (wj) calculados mediante (1) se normalizan por la suma, obteniendo la ponderación (wj estandarizada) de las variables. Ver tabla 3 
Tabla 3.

Matriz de correlaciones, desviaciones estándar y ponderaciones

\begin{tabular}{|l|c|c|c|c|c|c|c|c|c|c|}
\hline & $(\mathbf{1})$ & $\mathbf{( 2 )}$ & $\mathbf{( 3 )}$ & $\mathbf{( 4 )}$ & $\mathbf{( 5 )}$ & $\mathbf{( 6 )}$ & $\mathbf{( 7 )}$ & $\mathbf{( 8 )}$ & $\mathbf{w}_{j}$ & $\begin{array}{c}\mathbf{w}_{j} \\
\text { standardized }\end{array}$ \\
\hline (1) Trabajo & 1,000 & 0,401 & $-0,349$ & $-0,078$ & 0,482 & $-0,738$ & $-0,087$ & $-0,242$ & 3,011 & 0,128 \\
\hline (2)Coste capital físico & 0,401 & 1,000 & $-0,692$ & 0,271 & 0,743 & $-0,557$ & $-0,475$ & $-0,191$ & 2,721 & 0,116 \\
\hline (3)Coste depósitos/capital & $-0,349$ & $-0,692$ & 1,000 & $-0,036$ & $-0,941$ & 0,764 & $-0,012$ & $-0,018$ & 3,238 & 0,137 \\
\hline (4)ROA & $-0,078$ & 0,271 & $-0,036$ & 1,000 & 0,001 & 0,055 & $-0,709$ & $-0,817$ & 3,220 & 0,137 \\
\hline (5)Rent. Obt. dinero prest. & 0,482 & 0,743 & $-0,941$ & 0,001 & 1,000 & $-0,862$ & 0,086 & 0,121 & 2,649 & 0,112 \\
\hline (6)Tasa morosidad & $-0,738$ & $-0,557$ & 0,764 & 0,055 & $-0,862$ & 1,000 & $-0,219$ & 0,007 & 3,556 & 0,151 \\
\hline (7)Fondo cobertura & $-0,087$ & $-0,475$ & $-0,012$ & $-0,709$ & 0,086 & $-0,219$ & 1,000 & 0,775 & 2,599 & 0,110 \\
\hline (8)Solvencia/Ratio BIS & $-0,242$ & $-0,191$ & $-0,018$ & $-0,817$ & 0,121 & 0,007 & 0,775 & 1,000 & 2,566 & 0,109 \\
\hline Standard deviation & 0,396 & 0,363 & 0,391 & 0,387 & 0,360 & 0,416 & 0,340 & 0,348 & & \\
\hline
\end{tabular}

Leyenda: (1) Trabajo. (2) Coste del capital físico. (3) Coste depósitos/Capital. (4) ROA . (5) Rentabilidad obtenida por el dinero prestado. (6) Tasa de morosidad. (7) Fondo de cobertura. (8) Ratio BIS

Cuarto Paso. Ponderación de las empresas

Obtenidas las ponderaciones de cada criterio, aplicando (2) se obtiene la ponderación de las distintas entidades financieras.

Tabla 4.

Ponderación de las entidades financieras

\begin{tabular}{|c|c|c|c|c|c|c|}
\hline & CAM & Pastor & Bankinter & Sabadell & Banesto & Popular \\
\hline Ponderación & 0,482 & 0,411 & 0,476 & 0,435 & 0,387 & 0,432 \\
\hline
\end{tabular}

Quinto Paso. Cálculo del Ratio de Valoración

A pesar de que los bancos seleccionados como comparables son los más similares dentro de los cotizados en el mercado bursátil español, el rango que presenta el valor bursátil es muy elevado. Con el fin de homogeneizar la información del numerador del Ratio de Valoración, se ha optado por utilizar en lugar de dicho valor, una magnitud relativa como es el price-to-book ratio, cociente entre el valor bursátil medio durante 2009 y el patrimonio neto.

Como denominador del Ratio de Valoración, según (3), se utiliza la ponderación de las entidades financieras obtenida en el paso cuarto. 
Tabla 5.

Ratio price-to-book y ratio CRITIC

\begin{tabular}{|l|c|c|c|c|}
\hline & $\begin{array}{c}\text { Capitalización } \\
\text { bursátil media en } \\
\mathbf{2 0 0 9}(\boldsymbol{(})\end{array}$ & $\begin{array}{c}\text { Patrimonio Neto } \\
(\boldsymbol{\epsilon})\end{array}$ & P to B Ratio & Ponderación \\
\hline CAM & & 2.837 .237 .000 & - & 0,482 \\
\hline Pastor & $1.308 .968 .563,016$ & 1.610 .211 .000 & 0,813 & 0,411 \\
\hline Bankinter & $3.737 .675 .124,926$ & 2.583 .011 .000 & 1,447 & 0,476 \\
\hline Sabadell & $5.264 .075 .433,071$ & 5.297 .370 .000 & 0,994 & 0,435 \\
\hline Banesto & $5.407 .951 .190,048$ & 5.472 .536 .000 & 0,988 & 0,387 \\
\hline Popular & $7.837 .245 .800,617$ & 8.447 .984 .000 & 0,928 & 0,432 \\
\hline
\end{tabular}

Como resultado de aplicar (3)

$$
R V=\frac{0,813+1,447+0,994+0,988+0,928}{0,411+0,476+0,435+0,387+0,432}=2,414
$$

se obtiene un Ratio de Valoración RV=2,414.

Sexto Paso. Cálculo del valor de la CAM

Partiendo del Ratio de Valoración y del price-to-book ratio de la CAM se obtiene el valor de mercado por unidad de patrimonio neto.

Valor de mercado por unidad de patrimonio neto CAM $=2,414 * 0,482=1,163$

El producto del valor de mercado por unidad de patrimonio neto de la CAM por su patrimonio neto proporciona el valor bursátil de la CAM.

Valor Bursátil CAM $=1,163 * 2.837 .237 .000 €=3.301 .261 .437 €$

Una vez obtenido el valor teórico de la empresa, conviene validarlo. Con esta finalidad se ha procedido a calcular los precios de las acciones de cada una de las instituciones financieras utilizadas como comparables mediante el Ratio de Valoración obtenido. De esta manera se comprueba si los valores calculados de los comparables se encuentran dentro del rango de cotización de dichas acciones en el periodo septiembre de 2009 y el momento de la valoración, septiembre de 2010. Como puede observarse en la tabla 6, en todos los casos el precio calculado se encuentra dentro del rango de cotización. 
Tabla 6.

Valor por acción: cotización teórica vs. cotización real en el periodo septiembre 2009septiembre 2010

\begin{tabular}{|c|c|c|c|}
\hline & Valor Calculado $(\boldsymbol{\epsilon})$ & Min $(\boldsymbol{\epsilon})$ & Max $(\boldsymbol{\epsilon})$ \\
\hline Pastor & 4,980 & 3,100 & 6,024 \\
\hline Bankinter & 4,504 & 4,195 & 9,160 \\
\hline Sabadell & 3,668 & 2,970 & 5,111 \\
\hline Banesto & 5,764 & 4,930 & 9,534 \\
\hline Popular & 5,298 & 3,320 & 7,441 \\
\hline
\end{tabular}

\section{CONCLUSIONES}

En la actualidad, son numerosas las ocasiones en las que es necesario conocer el valor de las empresas. Las metodologías de valoración tradicionales constituyen un instrumento importante para el valorador aunque su aplicación puede presentar dificultades en algunos casos, por lo que la propuesta de nuevos métodos de valoración siempre es interesante. En ese sentido, lo que se pretende con nuestra propuesta es, precisamente, dotar al valorador de otro instrumento adicional y complementario que le permita abordar la valoración de empresas en situaciones de escasa información y acotar con mayor precisión el valor de una empresa problema.

En este trabajo, se ha presentado una nueva metodología basada en técnicas multicriterio que se enmarca dentro de los métodos comparativos de valoración o de enfoque de mercado. Se estructura en cinco pasos y utiliza el método multicriterio CRITIC y el Ratio de Valoración de las Normas Internacionales de Valuación. En primer lugar, se seleccionan las empresas comparables con la empresa problema, se determinan los criterios comparativos y se pasa a ponderar la importancia de estos criterios. Con ello, y mediante CRITIC, se calcula la ponderación de las empresas comparables y la que es objeto de valoración. Seguidamente, se calcula el Ratio de Valoración, de forma que el producto de este ratio por la ponderación de la empresa problema nos proporciona el valor de ésta.

Entre las aplicaciones más destacables cabe resaltar la valoración de empresas no cotizadas que, por su tipo de actividad y su tamaño son similares a otras que sí cotizan y para las que la capitalización bursátil representa el valor de mercado. Son numerosas las empresas que se encuentran en esta situación y cuya valoración puede resultar necesaria en un futuro inmediato. Un ejemplo lo presentan las cajas de ahorro, especialmente en el contexto actual, en el que es muy probable un cambio en su normativa reguladora que puede hacer necesario conocer su valor de mercado. Es precisamente esta circunstancia la que ha 
motivado que en este trabajo el ejemplo del funcionamiento de la nueva metodología se haya ilustrado a través de la valoración de una caja de ahorros, la Caja de Ahorros del Mediterráneo, CAM.

Finalmente, el presente trabajo no agota esta línea de investigación relacionada con la valoración multicriterio de empresas, más bien al contrario. De hecho, en el futuro, se pretende desarrollar el modelo incluyendo variables cualitativas mediante AHP.

AGRADECIMIENTOS: Los autores agradecen a dos revisores anónimos sus comentarios y sugerencias, que han permitido mejorar este trabajo.

\section{REFERENCIAS BIBLIOGRÁFICAS}

ALTMAN, E.I. (1968): "Financial ratios, discriminant analysis and the prediction of corporate bankruptcy", en The Journal of Finance, 23(4), pp.589-609.

ALTMAN, E.I. (1971): Corporate bankruptcy in America. Lexington-Mass: DC Heath \& Company. pp. (271).

ALTMAN, E.I. (1973): "Predicting railroad bankruptcies in America", en Bell Journal of Economics and Management Science, 4(1), pp. 184-211.

ALTMAN, E.I. (1993): Corporate financial distress and bankruptcy: A complete guide to predicting and avoiding distress and profiting from bankruptcy. New York: Wiley. pp. (368).

ARAGONÉS, P., AZNAR, J. FERRIS, J., GARCÍA-MELÓN, M. (2008).:"Valuation of urban industrial land: An analytic network process approach", en European Journal of Operational Research, 185(1), pp. 322339.

ARÉVALO-QUIJADA, M.T., GÓMEZ-DOMÍNGUEZ, D., VAZQUEZ-CUETO, M.J., ZAPATA-REINA, A. (2002): "Un estudio de las cajas de ahorros andaluzas mediante el método multicriterio promethee", en Revista de Estudios de Economía Aplicada, 20, pp. 5-27.

AZNAR, J., ESTRUCH, V. (2007): "Valoración de activos ambientales mediante métodos multicriterio. Aplicación a la valoración del Parque Natural del Alto Tajo", en Economía y Recursos Naturales, 7(13), pp. 107-126.

AZNAR, J., GUIJARRO, F. (2004): "Métodos de valoración basados en la programación por metas: modelos de valoración restringido", en Revista Española de Estudios Agrosociales y Pesqueros, 204, pp. 29-46. 
AZNAR, J., GUIJARRO, F. (2007a): "Estimating regression parameters with imprecise input data in an appraisal context", en European Journal of Operational Research, 176(3), pp. 1896-1907.

AZNAR, J., GUIJARRO, F. (2007b): "Modeling aesthetic variables in the valuation of paintings: an interval goal programming approach", en Journal of the Operational Research Society, 58, pp.957-963.

AZNAR, J., GUIJARRO, F., MORENO-JIMÉNEZ, J.M. (2007): "Valoración agrarian multicriterio en un entorno con escasa información", en Estudios de Economía Aplicada, 25(2), pp. 549-572.

AZNAR, J., GUIJARRO, F. MORENO-JIMÉNEZ, J.M. (2008): "Mixed valuation methods: a combined AHP-GP procedure for individual and group multicriteria agricultural valuation", en Annals of Operations Research, (en prensa), DOI 10.1007/s10479-009-05272-2.

BEAVER, W.H. (1966): "Financial ratios as predictors of failure", en Empirical Research in Accounting: Selected studies, 4, pp.71-111.

BEAVER, W.H. (1968): "Alternative accounting measures as predictors of failure", en Accounting Review of Accounting Studies, 1, pp.267-284.

CERVELLÓ ROYO, R., GARCÍA GARCÍA; F., GUIJARRO MARTÍNEZ, F. (2010): "Ranking multicriterio de viviendas: una adaptación del modelo de precio único", en Revista de Estudios de Economía Aplicada, 28 (3), en prensa.

DENG, H., YEH, CH., WILLIS, R.J. (2000): "Inter-company comparison using modified TOPSIS with objective weigts", en Computers \& Operations Research, 27, pp.963-973.

DIAKOULAKI, D., MAVROTAS, G., PAPAYANNAKIS, L.(1995): "Determining objective weights in multiple criteria problems: The critic method", en Computers \& Operations Research, 27, pp. 963-973.

FENG, CH. M., WANG, R.T.(2001): "Considering the financial ratios on the performance evaluation of highway bus industry", en Transport Reviews, 21(4), pp.449-467.

GARCIA, F., GUIJARRO, F., MOYA, I. (2008): "La valoración de empresas agroalimentarias: una extensión de los modelos factoriales", en Revista de Estudios Agrosociales y Pesqueros, 217, pp. 155-181.

GARCIA, F., GUIJARRO, F., MOYA, I. (2010a): "Factores financieros clave en la reorganización del sector de las cajas rurales", en CIRIEC-Revista de Economía Pública, Social y Cooperativa, 68, pp.61-79.

GARCIA, F., GUIJARRO, F., MOYA, I. (2010b): "A goal programming approach to estimating performance weights for ranking firms", en Computers and Operations Research, 37, pp. 1597-1609.

GARCIA-CESTONA, M., SURROCA, J. (2006): "Evaluación de la eficiencia con múltiples fines. Una aplicación a las cajas de ahorro", en Revista de Economía Aplicada, 40, pp.67-89. 
GARCIA-MELÓN, M., FERRÍS-OÑATE, J., AZNAR-BELLVER, J., ARAGONÉSBELTRÁN, P., POVEDA-BAUTISTA, R.(2008): "Farmland appraisal based on the Analytic network process", en Journal of Global Operational, 42, pp. 143-155.

GUIJARRO, E., GUIJARRO, F. (2010): "Valoración multicriterio de empresas: una aplicación al sector bodeguero español", en Revista de Estudios Agrosociales y Pesqueros, en prensa.

HALKOS, G.E., SALAMOURIS, D.S.(2004): "Efficiency measurement of the Greek commercial banks with the use of financial ratios: a data envelopment analysis approach", en Management Accounting Research, 15, pp.201-224.

INTERNATIONAL VALUATION STANDARDS COMMITTEE. (2007): International Valuation Standards, IVS. IVSC: London.

LI, H., ADELI, H., SUN, J., HAN, J.G. (2010): "Hibrydizing principles of TOPSIS with case based reasoning for business failure prediction", en Computers and Operations Research, 38(2), pp. 409-419.

MALHOTRA, R., MALHOTRA, D.K., LAFOND, A. (2009): "Analysing financial services industry using data envelopment analysis", en International Journal of Applied Management Science, 1(3), pp.217-246.

MIRALLES-MARCELO, J.L., MIRALLES-QUIRÓS, J.L. (2002): "Factores determinantes del valor bursátil de las empresas portuguesas (1991-1999). Nuevas propuestas metodológicas", en Revista Española de Financiación y Contabilidad, 112, pp.495-528.

MOYA, I. (1995): "Valoración bursátil de empresas. Propuesta de una metodología", en Análisis Financiero, 66, pp.92-106.

MOYA, I. (1996): "Valoración analógico-bursátil de empresas. Aplicación a las cajas de ahorro", en Revista Española de Financiación y Contabilidad, 25 (86), pp.199-234.

KUMBHAKAR, S.C., LOZANO-VIVAS, A., LOVELL, C.A.K., Hasan, I. (2001): "The effects of deregulation on the performance of financial institutions: The case of Spanish savings banks", en Journal of Money Credit Banking, 33, pp.101-120.

OHLSON, J.A.(1980): "Financial ratios and the probabilistic prediction of bankruptcy", en Journal of Accounting Research, 18(1), pp.109-131.

PISILLAKI, M., TSOLAS, I.E., MARGARITIS, D. (2010): “Evaluation of credit risk based on firm performance", en European Journal of Operational Research, 201, pp.873-881.

PRIOR, D. (2003): " Long and short-run nonparametric cost frontier efficiency: An application to Spanish saving banks", en Journal of Banking and Finances, 27, pp.107-123.

SUN, L., SHENOY, P.P. (2007): "Using Bayesian networks for bankruptcy prediction: some methodological issues", en European Journal of Operational Research, 180(2), pp.738-753. 
VIDAL-GIMÉNEZ, F., SALES-CIVERA, J.M., LÓPEZ-LLUCH, D.B. (2004): "LoS modelos de valoración de empresas: aplicación de los modelos dinámicos de valoración analógico-bursátil a las cooperativas agrarias", en Spanish Journal of Agricultural Research, 2(1), pp.17-25

WANG, Y.L., LEE, H.S. (2008): "A clustering method to identify representative financial ratios", en Information Sciences, 178, pp. 1087-1097.

YEH, Q.J. (1996): "The application of data envelopment analysis in conjunction with financial ratios for bank performance evaluation", en Journal of the Operational Research Society, 47, pp.980-988. 
